Short report

\title{
Sofosbuvir/velpatasvir in treatment-experienced HCV-infected patients - short report
}

\author{
Anna Piekarska', Aleksandra Berkan-Kawińska', Zbigniew Deroń2, Justyna Ciupińska², Jolanta Białkowska \\ 'Department of Infectious Diseases and Hepatology, Medical University of Lodz, Poland \\ 2Department of Infectious Diseases and Liver Diseases, WSSz Bieganski, Lodz, Poland
}

\begin{abstract}
Aim of the study: The aim of this overview was to evaluate the efficacy of sofosbuvir/velpatasvir (SOF/VEL) combination in a real-life setting, with particular regard to treatment-experienced individuals.

Material and methods: Seventy-five consecutive patients who were treated with SOF/VEL, completed the 12-week follow-up and had sustained virologic response (SVR) evaluated were included in the analysis. Out of them, $60(80 \%)$ patients were treatment-naïve and $15(20 \%)$ were treatment-experienced.

Results: SVR rates reached $89.4 \%$ (66/75) in the whole study group and were comparable irrespective of the fibrosis stage or HCV genotype. However, a significant difference in treatment efficacy between treatment-naive and treatment-experienced individuals was observed, with SVR rates of $98.3 \%(59 / 60)$ and $46.7 \%(7 / 15)$, respectively $(p<0.0001)$.

Conclusions: Further studies including large real-life cohorts of treatment-experienced patients treated with SOF/VEL are warranted to elucidate the real efficacy of this regimen as a retreatment option.
\end{abstract}

Key words: retreatment, chronic hepatitis $C$, velpatasvir/sofosbuvir.

\section{Address for correspondence}

Prof. Anna Piekarska, Department of Infectious Diseases and Hepatology, Medical University of Lodz, Poland,

e-mail: annapiekar@gmail.com

\section{Introduction}

High efficacy of interferon-free (IFN-free) directacting antiviral (DAA) therapies has led to a major breakthrough in hepatitis $\mathrm{C}$ virus (HCV) care. Cure rates in treatment-naïve individuals reached up to $95 \%$ [1]. Moreover, numerous clinical and observational trials have demonstrated comparable sustained virologic response (SVR) rates irrespective of treatment status, and history of treatment failure did not prove to be associated with decreased DAA efficacy [2-5]. Combination of sofosbuvir/velpatasvir (SOF/VEL), which includes a NS5B nucleotide polymerase inhibitor that displays a high barrier to resistance and excellent antiviral activity, is particularly recommended for individuals with a history of treatment failure [6]. It has been addressed in EASL 2018 guidelines as a main retreatment option for treatment-experienced patients with genotype 1, 2, 4, 5 and 6 [7]. The aim of the following overview was to evaluate the efficacy of SOF/ VEL combination in a real-life setting with particular regard to treatment-experienced individuals.

\section{Material and methods}

Our retrospective analysis included all patients who were treated with SOF/VEL in Biegański Hospital (Lodz, Poland) in the years 2018-2019. Eligible individuals who adhered to treatment requirements were linked to the National Hepatitis C Treatment Programme and received the treatment. Seventy-five consecutive patients who were treated with SOF/VEL, completed the 12-week follow-up and had SVR evaluated were included in the study. There were 40 (53\%) men and 35 (47\%) women aged 18-84 years. Out of 
them, $60 / 75(80 \%)$ were treatment-naïve and 15/75 (20\%) were treatment-experienced.

Treatment-naïve individuals received a single pill combination of SOF/VEL for 12 weeks. Treatment-experienced patients received the same dose of SOF/VEL and were treated for 12 (12 patients) or 24 weeks (3 patients). In the last two cases ribavirin (RBV) in the dose of $1000 \mathrm{mg}$ daily was added. Treatment-experienced patients treated with SOF/VEL \pm RBV for 24 weeks were previously treated with SOF/RBV for 24 weeks (one patient), pegylated IFN (PegIFN) and SOF/ledipasvir (SOF/LDV) for 12 weeks (one patient), and finally with PegIFN, ombitasvir-paritaprevir-ritonavir plus dasabuvir for 12 weeks and SOF/LDV for 12 weeks (one patient). The baseline characteristics of the study population are presented in Table 1.

Statistical analyses were performed with STATISTICA 13.1 (StatSoft, USA). Ethical approval was not necessary for this retrospective, observational study conducted in a real-life setting with approved drugs. Patient data were collected and analyzed according to the applicable personal data protection principles. Due to the retrospective character of the analysis no target sample size was planned. Median with interquartile range (IQR) and proportions were used for descriptive statistics, as appropriate. To compare the differences between groups the chi-square test was used (dichotomous variables). A $p$-value $<0.05$ was considered as statistically significant.

\section{Results}

The overall SVR rate in the whole study population of patients treated with SOF/VEL was $89.4 \%$ (66/75 patients). When compared in terms of liver fibrosis, patients with fibrosis F0-F2 achieved SVR in 90.9\% (20/22 patients), whereas patients with advanced fibrosis or liver cirrhosis (F3-F4) achieved SVR in $86.8 \%$ $(46 / 53)$. The difference did not prove to be statistically significant $(p>0.05)$. SVR rates in patients infected with genotype 1 or 4 (90\%; 36/40 patients) were slightly higher compared to patients infected with genotype 3 (85.7\%; 30/35 patients), but again the difference did not reach statistical significance $(p>0.05)$. However, SOF/VEL treatment efficacy was significantly worse in treatment-experienced patients compared to treatment-naïve patients, with SVR rates of $46.7 \%(7 / 15)$ and $98.3 \%(59 / 60)$, respectively $(p<0.0001)$ (Fig. 1).

Three out of eight treatment-experienced patients who failed SOF/VEL therapy were treated for 24 weeks $\pm \mathrm{RBV}$, as two of them failed previous NS5A inhibitor-based treatment. Four out of eight patients were treated with SOF/VEL for 12 weeks, since they had no
Table 1. Baseline characteristics of the study population

\begin{tabular}{ll}
\hline Characteristics & \\
\hline All patients & $75(100 \%)$ \\
\hline Male sex, $n(\%)$ & $40(53.3 \%)$ \\
\hline Age, range & $18-84$ years \\
\hline F3-4 fibrosis, $n(\%)$ & $53(70.7 \%)$ \\
\hline Genotype 3, $n(\%)$ & $35(46.7 \%)$ \\
\hline Treatment-experienced, $n(\%)$ & $15(20.0 \%)$ \\
\hline HIV coinfection, $n(\%)$ & $19(25.3 \%)$ \\
\hline
\end{tabular}

history of NS5A inhibitor failure (received PegIFN, SOF and RBV in the past). One patient with history of NS5A inhibitor failure (8 weeks of LDV/SOF) was treated with SOF/VEL for 12 weeks.

\section{Discussion}

This retrospective analysis of SOF/VEL combination efficacy in the real-life population demonstrated an overall SVR rate of $89.4 \%$, which is below the expectations for the modern DAA treatment regimens. The SVR rate was particularly low in the treatment-experienced group (46.7\%) and it was significantly worse than the SVR rate in treatment-naïve patients. The importance of this observation, though limited by the small sample size, puts into question the current HCV treatment guidelines which recommend SOF/VEL combination for patients with a history of DAA failure. Out of eight patients who experienced relapse, in only one who received 12 weeks of SOF/VEL the duration of therapy could have been potentially prolonged up to 24 weeks, since this patient had already failed 8 weeks of LDV/SOF in the past. The other seven patients were treated with the SOF/VEL regimen in accordance with

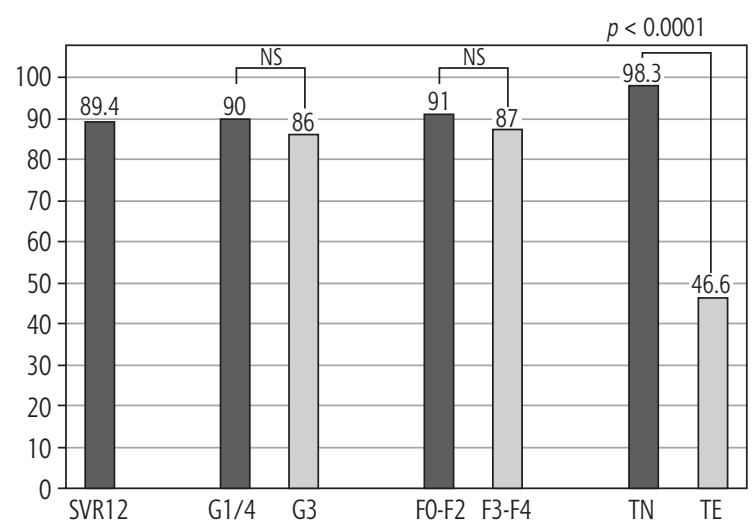

NS - not significant

Fig. 1. SOF/VEL treatment results - SVR rates overall and according to HCV genotype (1 and 4 vs. 3), fibrosis stage (F0-F2 vs. F3-F4) and treatment history (treatment-naïve [TN] vs. treatment-experienced [TE]) 
the summary of product characteristics, taking into account the history of former NS5A inhibitor-based failures. Even so, the therapy with SOF/VEL did not result in the achievement of SVR. Since in none of the cases was RAS testing performed before initiation of the treatment, we were not able to determine HCV drug resistance. Nevertheless, the failure rate of 8 per 15 patients is surprisingly high and has not been described to date.

Moreover, efficacy of the SOF/VEL regimen in patients with advanced fibrosis or genotype 3 infection (SVR rates of $86.8 \%$ and $85.7 \%$, respectively) is also among the lowest reported [8]. In a study by Mangia et al. the SVR rate achieved in patients with liver cirrhosis and genotype 3 after SOF/VEL therapy was over $97 \%$, while in the formerly published registrational trials it reached over $95 \%$ in patients infected with genotype 3 [9] and $94 \%$ in patients with decompensated liver cirrhosis [10]. It has to be noted that in our study out of 8 patients who failed SOF/VEL treatment, six had fibrosis F4 and five were infected with genotype 3. Therefore it may seem that such an unfortunate combination of poor prognostic factors (liver cirrhosis, genotype 3 , retreatment) might have negatively influenced the treatment efficacy. To date, however, neither advanced fibrosis nor history of treatment failure was associated with lower SVR rates after the SOF/VEL regimen. Nevertheless, it seems that in patients who are infected with genotype 3 , have liver cirrhosis and have already failed previous antiviral therapy, SOF/ VEL \pm RBV combination should no longer be considered a retreatment option and new DAA must be used. This issue has also been addressed in the EASL guidelines $[7,11]$.

The main limitation of our analysis is the small sample size. This is particularly true for treatment-experienced patients, who constituted merely $20 \%$ of the study population (15 subjects). However, the surprisingly low SVR rates achieved in this specific group motivated us to present the results as a short report. Further studies including large real-life cohorts of treatment-experienced patients treated with SOF/VEL are warranted to elucidate the real efficacy of this regimen as a retreatment option.

\section{Disclosure}

The authors report no conflict of interest.

\section{References}

1. Pisaturo M, Russo A, Onorato L, et al. Efficacy of 12-weeks velpatasvir plus sofosbuvir-based regimen in HCV-naive subjects with mild fibrosis: a meta-analysis. Acta Biomed 2019; 90: 187-196.
2. Bourlière M, Gordon SC, Flamm SL, et al. Sofosbuvir, velpatasvir, and voxilaprevir for previously treated HCV infection. N Engl J Med 2017; 376: 2134-2146.

3. Feld JJ, Jacobson IM, Hézode C, et al. Sofosbuvir and velpatasvir for HCV genotype 1, 2, 4, 5, and 6 infection. N Engl J Med 2015; 373: 2599-2607.

4. Gane EJ, Shiffman ML, Etzkorn K, et al. Sofosbuvir-velpatasvir with ribavirin for 24 weeks in hepatitis $\mathrm{C}$ virus patients previously treated with a direct-acting antiviral regimen. Hepatology 2017; 66: 1083-1089.

5. Pianko S, Flamm SL, Shiffman ML, et al. Sofosbuvir plus velpatasvir combination therapy for treatment-experienced patients with genotype 1 or 3 hepatitis $C$ virus infection: a randomized trial. Ann Intern Med 2015; 163: 809-817.

6. Pérez AB, Chueca N, García-Deltoro M, et al. High efficacy of resistance-guided retreatment of HCV patients failing NS5A inhibitors in the real world. J Hepatol 2019; 7: 876-888.

7. EASL recommendations on treatment of hepatitis C 2018. J Hepatol 2018; 69: 461-511.

8. Mangia A, Piazzolla V, Giannelli A, et al. Correction: SVR12 rates higher than $99 \%$ after sofosbuvir/velpatasvir combination in HCV infected patients with F0-F1 fibrosis stage: A real world experience. PLoS One 2019; 14: e0223287.

9. Foster GR, Afdhal N, Roberts SK, et al. Sofosbuvir and velpatasvir for HCV genotype 2 and 3 infection. N Engl J Med 2015; 373: 2608-2617.

10. Curry MP, O’Leary JG, Bzowej N, et al. Sofosbuvir and velpatasvir for $\mathrm{HCV}$ in patients with decompensated cirrhosis. $\mathrm{N} \mathrm{Engl}$ J Med 2015; 373: 2618-2628.

11. Naggie S. Treating HCV infection: it doesn't get much better than this. Top Antivir Med 2019; 26: 104-108. 